\title{
Dupuytren's disease digital radius IV right hand and carpal tunnel syndrome on ipsilateral hand
}

${ }^{1}$ UMF Carol Davila, Emergency Clinical Hospital Bucharest-Department of Plastic Surgery,Estetic and Microurgery

${ }^{2}$ University "Ovidius" of Constanta, Faculty of medicine

\begin{abstract}
Dupuytren's contracture is a fibroproliferative disease whose etiology and pathophysiology are unclear and controversial. It is a connective tissue disorder, which takes part in the palmar's fibromatosis category and has common characteristics with the healing process. Dupuytren's disease is characterized by the flexion contracture of the hand due to palmar and digital aponevrosis. It generally affects the 4th digital radius, followed by the 5 th one. Without surgery, it leads to functional impotence of those digital rays and/or hand. It is associated with other diseases and situational conditions like Peyronie's disease, the Lederhose disease (plantar fibromatosis), Garrod's digital knucklepads, diabetes , epilepsy, alcoholism, micro traumatisms, stenosing tenosynovitis and not the least with carpal tunnel syndrome. The carpal tunnel syndrome is a peripheral neuropathy with the incarceration of the median nerve at the ARC level, expressed clinically by sensory and motor disturbances in the distribution territory of the median nerve, which cause functional limitations of daily activities of the patient. After the failure of the nonsurgical treatment or the appearance of the motor deficit, is established the open or endoscopic surgical treatment with the release of the median nerve.
\end{abstract}

Bogdan Caraban

University "Ovidius" of Constanta, Faculty of medicine

Address: Universitatii Street, No. 1, Campus B, Constanta email: bcaraban@yahoo.com
Postoperative recovery in both diseases is crucial to the functionality of the affected upper limb and to the quality of the patient's life. The patient, a 61 years old man, admitted to the clinic for the functional impotence of the right hand, for the permanent flexion contracture of the metacarpophalangeal joint (MCP) and proximal interphalangeal joint (PIP) of the 4th finger with extension deficit, for the damage of the thumb pulp clamp of the 4th finger, for nocturnal paresthesia of fingers I-III and pain that radiates into the fingertips. After clinical, paraclinical, imagistic and electrical investigations, surgery is practiced partial aponevrectomy, carpal ligament section, external neurolysis of the median nerve, flexor tendon tenolisys. The particularity of this case is the coexistence of two pathologies: Dupuytren's disease and carpal tunnel syndrome, the decision to solve in the same operator time and the problem of immobilization. Reportation of this case supports previous reports in literature, such as Dupuytren's disease and carpal tunnel syndrome are observed at the same patient, at the same time or one after another.

Keywords: Dupuytren disease, carpal tunnel syndrome

\section{Introduction}

Dupuytren's contracture [1-5] is a fibroproliferative disease whose etiology and pathophysiology are unclear and controversial. It is a connective tissue disorder, which takes part in the 
palmar's fibromatosis category and has common characteristics with the healing process. Dupuytren's disease is characterized by the flexion contracture of the hand due to palmar and digital aponevrosis. It generally affects the 4th digital radius, followed by the 5th one. Without surgery, it leads to functional impotence of those digital rays and/or hands. It is associated with other diseases and situational conditions like Peyronie's disease, the Lederhose disease (plantar fibromatosis), Garrod's digital knukle-pads, diabetes, epilepsy, alcoholism, micro traumatisms, stenosing tenosynovitis and not the least with carpal tunnel syndrome [6-19].

The carpal tunnel syndrome is a peripheral neuropathy with the incarceration of the median nerve at the ARC level, expressed clinically by sensory and motor disturbances in the distribution territory of the median nerve, which cause functional limitations of daily activities of the patient. After the failure of the nonsurgical treatment or the appearance of the motor deficit, is established the open or endoscopic surgical treatment with the release of the median nerve [2029].

Postoperative recovery in both diseases is crucial to the functionality of the affected upper limb and to the quality of the patient's life.

\section{Gase presentation}

The patient, a 61 years old man, pensioner, is admitted in the Clinic of Plastic Surgery, Aesthetic and Reconstructive Microsurgery at the Emergency Hospital Bucharest, "Floreasca" for the functional impotence of the right hand, for the permanent flexion contracture of the metacarpophalangeal joint (MCP) and proximal interphalangeal joint (PIP) of the 4th finger with extension deficit, for the damage of the thumb pulp clamp of the 4th finger, for nocturnal paresthesia of fingers I-III and pain that radiates into the fingertips.

From personal pathological history, is observed that the patient suffered from type II diabetes, in treatement with OAD and as conditions of life and work he was a professor of needlework (keep in mind the possibility of existence of micro traumatisms ).

\section{Affection history}

The onset is insidious, with 3 years ago, with no history of local trauma .

The period of state consists in the appearance of the first distal palmar crease nodule formation of a contractile straps at finger IV , nocturnal paresthesias about 2-3 months. Evolved to worsening symptoms .

Current Status : paresthesia, palmar contracture out across digital radius IV without established diagnosis or treatment followed regarding this symptomatology

Local examination revealed normal skin colored , slender ; adherent skin deep plan in the radius IV; a nodule and a longitudinal cord on the palmar side of the radius IV from the distal palmar flexion crease over MPC and prolonged F1-F2; affecting the normal alignment of the fingers at rest and full extension; not found muscular atrophy ; trophicity skin, sweating and normal T; Tinel sign $(+)$ at radiocarpal joint with pain that radiates to the apical level finger II - III of the right hand; Phalen test $(+)$ patient describing " tingling " apical level finger I- III; carpal compression test (Durkan) $(+)$; discriminative test in 2 points $(-)$; revealed mild tenderness hypoesthesia in territory median nerve; lightweight fine movements of the right hand disorders; capillary pulse current.(Figure $1-2)[2,5,7,14]$

Rx does not reveal osteoarticular changes, but serves for differential diagnosis in both diseases.

Ultrasonography can decrease thickening of the palmar fascia, presence of nodules and cords, location flexor tendons, changes of the median nerve caliber through the carpal tunnel $[23,24]$.

EMG is done in the short abductor thumb median nerve proximal to the carpal tunnel ex: flexor carpi radialis muscles, round pronator, long 
thumb flexor, excluded proximal median neuropathy .muscle to detect early changes in the median nerve denervation. [1-5]

EMG can test for differential diagnosis:

-Two or more muscles innervated by the

-Two or more muscles innervated C6- C7 ex: round pronator, triceps, extensor fingers, cervical radiculopathy excluded .

-Two or more muscles innervated C8 -T1 egg I dorsal interosseous, extensor thumb, excluded a brachial plexopathy, polyneuropathy and C8 -T1 radiculopathy.



Figure 1. [30]

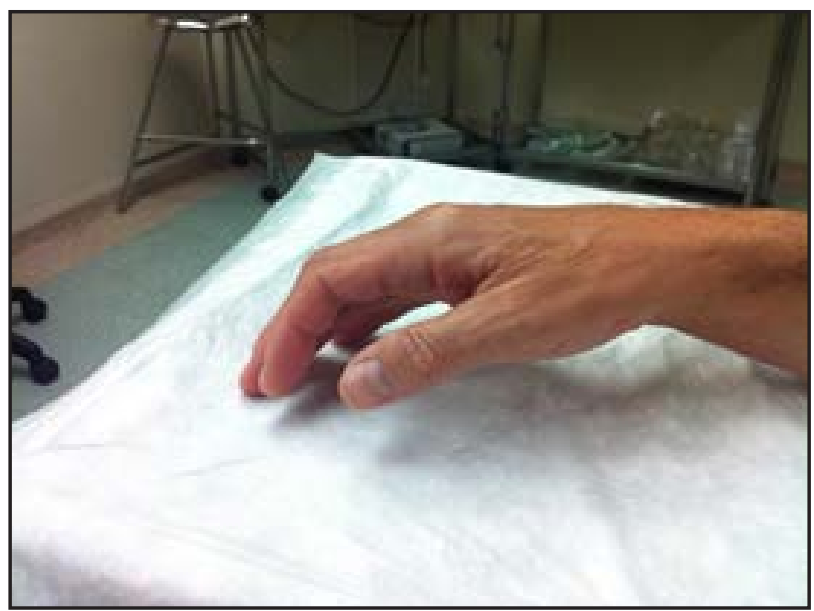

Figure 2. [30]

Differential diagnosis in Dupuytren's disease is made with : sequelae scar retraction of the hand with flexion after various injuries : burns, wounds, bruises, with damage of skin and / or tendon, bone, ligament; infiltrative tumor formations : giant cell tumor of soft parts, epitheloid sarcoma ; intrinsic joint contracture : nerve damage or ischemia ( $\mathrm{sdr}$ Volkmann ); palmar ganglions; epidermoid inclusion cyst; stenosis or nodular tenosynovitis digitopalmare ; changes secondaries rheumatoid arthritis ; occupational hyperkeratosis ; callus [1-19].

Differential diagnosis in the carpal tunnel syndrome is made with: proximal nerve damage ; hypertrophic arthritis ; compression of the median nerve in the round pronator muscle; neuritis associated with local corticosteroid therapy ; progressive muscular atrophy; ulnar neuropathy [1-5,20-29].

Evolution in the absence of treatment is to emphasize digital flexion, and the other fingers catching, affecting aponeurosis in total, with appearance of joint changes, trophic and sensitivity disorders.

* can be found in the literature and description of rare spontaneous regression of DD

Conservative treatment of DD:

- treatments without semnificative results: immobilization, physiotherapy, ultrasound, radiation therapy, medication antigout creams with vitamin $\mathrm{E}$ [6-19].

- treatments with semnificative results: percutaneous needle fasciotomy, steroid injections , continuous skeletal traction light, gamma interferon, vitamin E, enzyme percutaneous fasciotomy [6-19].

Conservative treatment CTS: orthopedic local corticosteroid injections, physiotherapy, a possible change in the workplace [20-29].

\section{Surgical treatment [1-29]}

Based on the information obtained, consider with the patient the optimal time for surgery of both diseases. Medical treatment is taken in discussion as well as preoperative preparation postoperative treatment .Surgery is the only one able to bring rapid relief of symptoms of both diseases, restore hand function, elimination and prevention of contractures 
and their recurrence (Dupuytren's d. 20-80 \% relapse) , removal the risk of irreversible damage or modest possibilitiestoimprove function hand (Full contracture , amitrophy ) .

The risk is medium -scale operator ADRIANI MOORE scheduled for surgery, 3 .

After preoperative and anesthesial preparation is practiced surgery.

\section{Tehnical surgery}

It was chosen longitudinal incision digital palm type Bruner centered on the 4th radius.which starts from the distal palmar crease to AIFD, with a fold extension of the thumb opposition to the insertion of long palmar tendon.(Figure 3) It performs dissection and off of skin, emphasizing the cord and the nodule. (Figure.4)

It makes regional aponevrectomie (subtotal) with removal aponeurosis affected by longitudinal dissection with cord and nodule excision.(Figure 5)

It mobilizes finger IV, highlighting the tendons and joint mobility and integrity and fluidity of digital nerve and vessel. Incision continue until radiocarpal joint with carpal ligament sectioning and aponevrotomie; the median nerve is isolated, the carpal tunnel is explored with obvious causes compression removal.(Figures 6-8) The external neurolysis and tenolisys flexor tendons is praacticed, with the removal of thickened sheats, lavage, hemostasis, drainage aspiration, suturing, dressing, immobilization.(Figure 9,10)

Postoperative suction drainage suppress after 2 days, antibiotic therapy is instituted, painkiller, NSAIDs, antisecretory and cold local applications. Start of active and passive movements of MCP at 3 days after surgery, immobilization of PIP at night. Maintaining immobilization 14 days.

Follow rehabilitation therapy program.

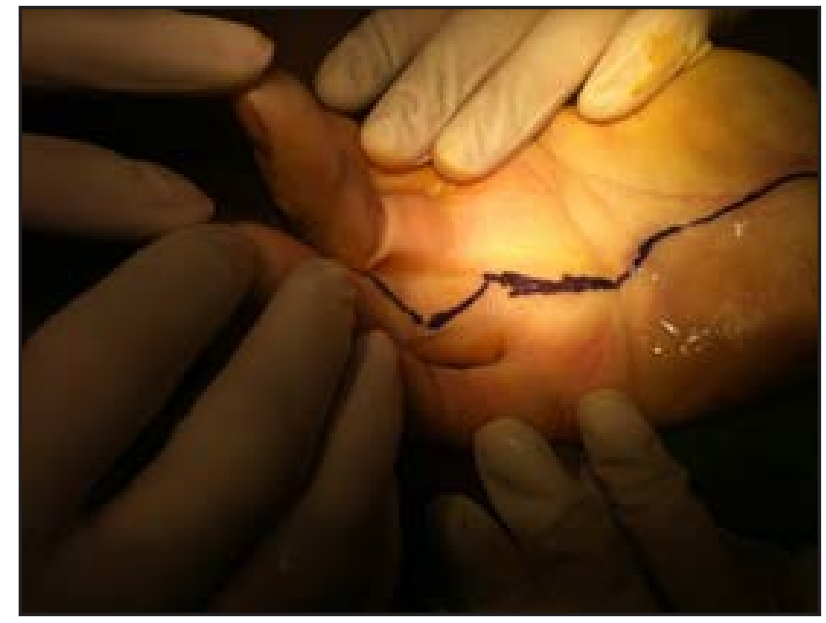

Figure 3. [30]

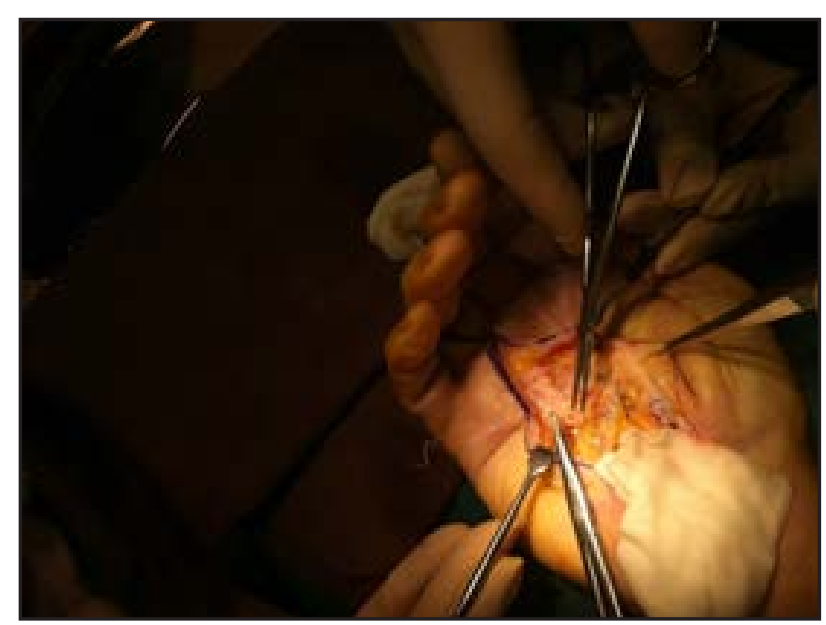

Figure 4. [30]

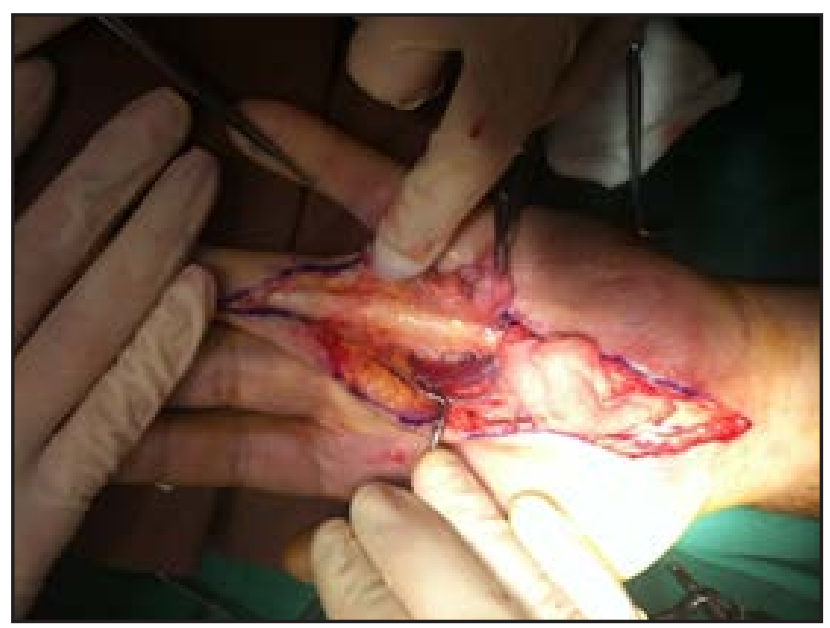

Figure 5. [30] 


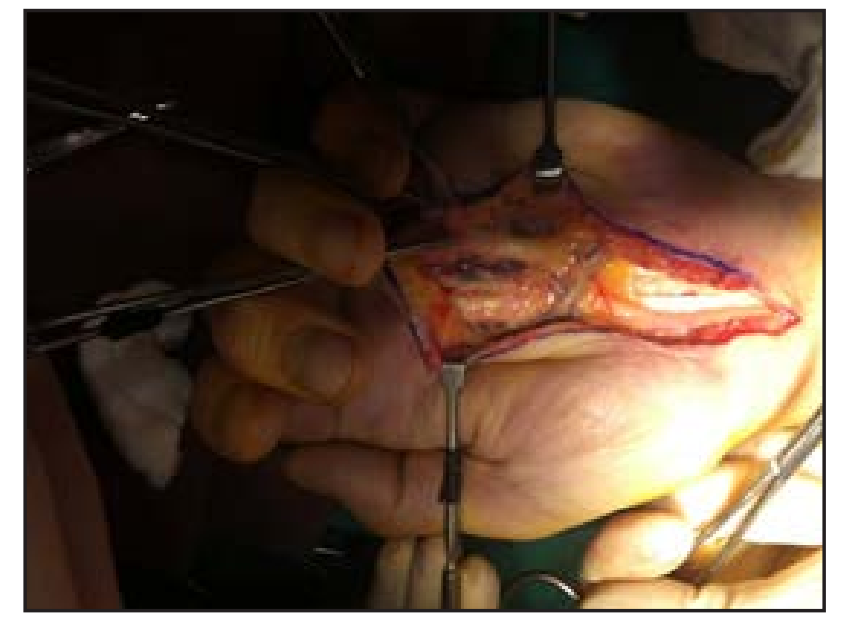

Figure 6. [30]

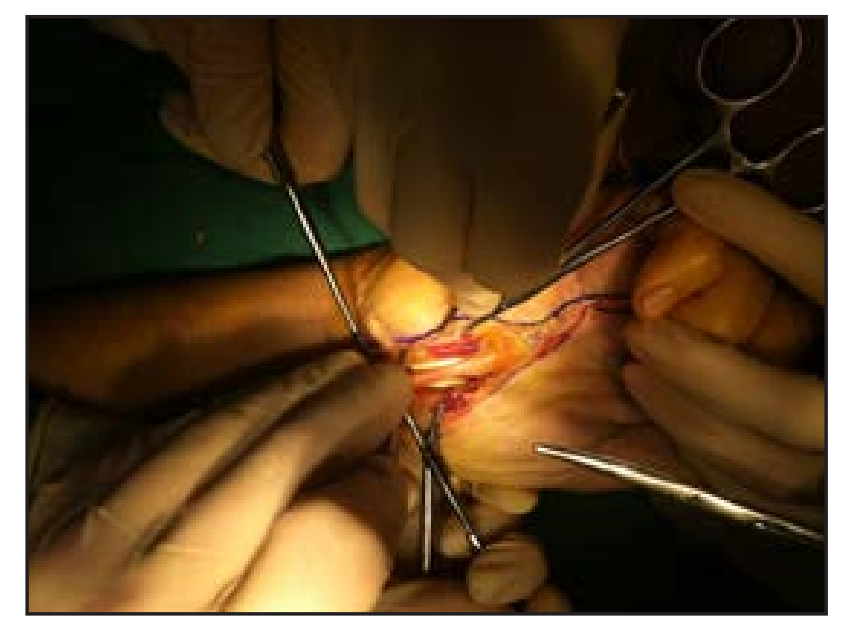

Figure 7. [30]

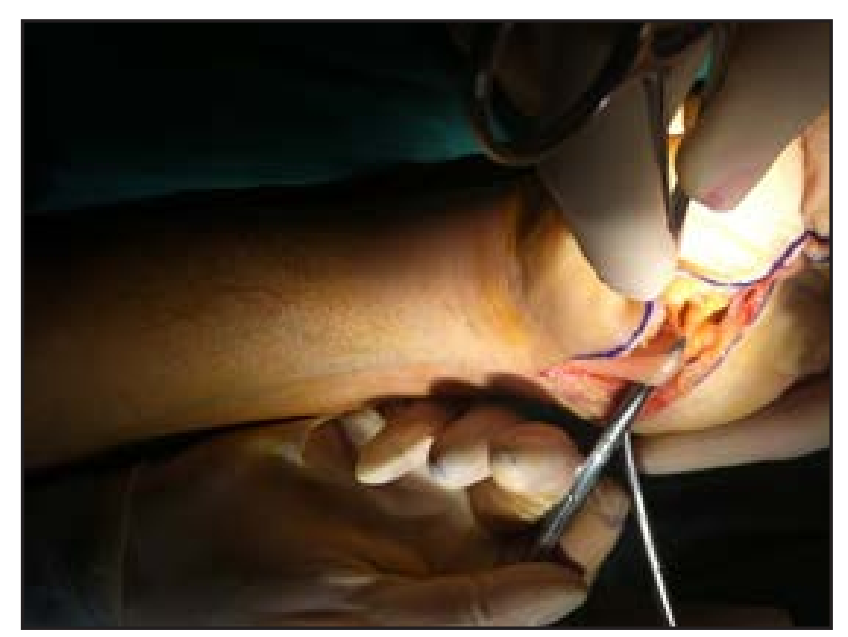

Figure 8. [30]



Figure 9. [30]

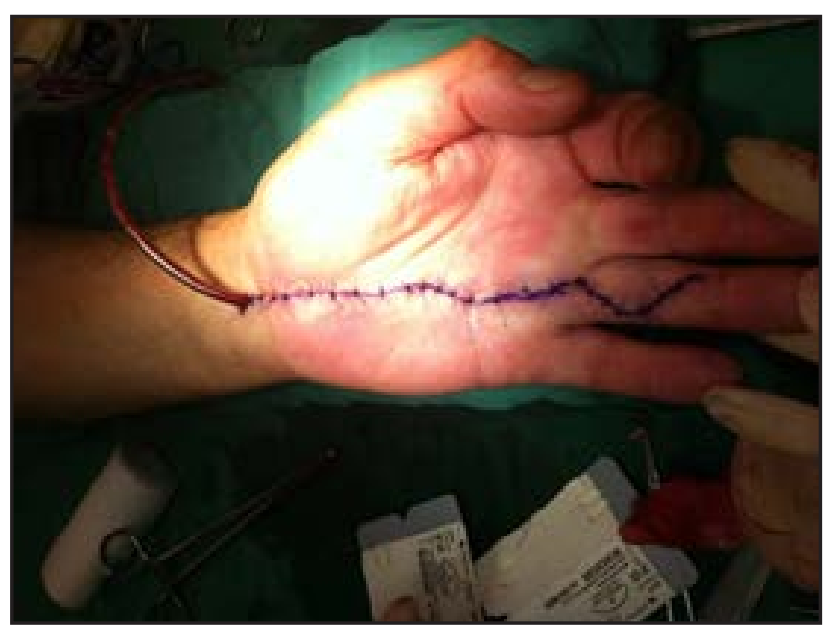

Figure 10. [30]

At 3 months highlight the possibility of performing MCP and PIP joint extension, absence of pain, numbness and resumption of daily activities. (Figure 11) 


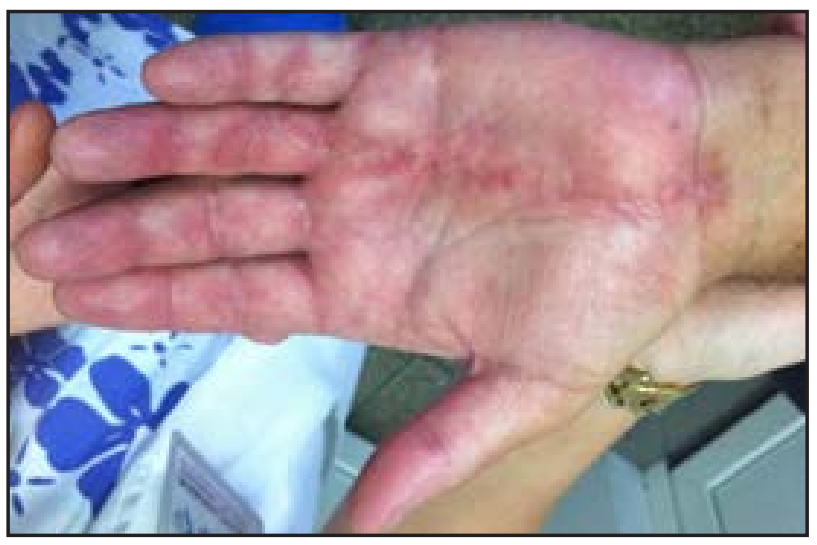

Figure 11. [30]

\section{Prognostic and evolution:}

This case has a good prognosis both early and late (with attention to relapses DD)

\section{Conclusions and discussions}

The particularity of this case is the coexistence of two pathologies: Dupuytren's disease and carpal tunnel syndrome, the decision to solve in the same operator time and the problem of immobilization. Reportation of this case supports previous reports in literature, such as Dupuytren's disease and carpal tunnel syndrome are observed at the same patient, at the same time or one after another.

\section{Acknowledgments}

„This paper was co-financed from the European Social Fund, through the Sectorial Operational Programme Human Resources Development 2007-
2013, project number POSDRU/159/1.5/S/138907 "Excellence in scientific interdisciplinary research, doctoral and postdoctoral, in the economic, social and medical fields -EXCELIS", coordinator The Bucharest University of Economic Studies".

\section{References}

1. Leclercq C. (2000). Epidemiology. In Tubiana R., Leclercq C., Hurst L.C., Badalamente M.A. \& Mackin E.J. (Eds.). Dupuytren's disease (pp. 53-58). London: Martin Dunitz.

2. Cederlund R.I., Thomsen N., Thrainsdottir S., Eriksson K.F., Sundkvist G. \& DahlinL .B. (2009). Hand disorders, hand function, and activities of daily living in elderly men with type 2 diabetes. J Diabetes Complications. 23(1), 32-39.

3. Leclercq C. (2000) Treatment. In Tubiana R., Leclercq C., Hurst L.C., Badalamente M.A. \& Mackin E.J. (Eds.). Dupuytren's disease (pp. 204-222). London: Martin Dunitz.

4. Leclercq C. (2000) Results of surgical treatment. In Tubiana R., Leclercq C., Hurst L.C., Badalamente M.A. \& Mackin E.J. (Eds.). Dupuytren's disease (pp. 239-249). London: Martin Dunitz.

5. Gelberman R.H. (1991). Operative nerve repair and reconstruction. Philadelphia:J.B. Lippincott Company.

6. Wilburn J., McKenna S..P, Perry-Hinsley D. \& Bayat A. (2013). The impact of Dupuytren disease on patient activity and quality of life. $J$ Hand Surg Am. 38(6),1209-1214.

7. Dolmans G.H., de Bock G.H. \& Werker P.M. (2012). Dupuytren diathesis and genetic risk. $J$ Hand Surg Am. 37(10), 2106-2111.

8. van Rijssen A.L., ter Linden H., Werker P.M. (2012) Five-year results of a randomized clinical trial on treatment in Dupuytren's disease: percutaneous needle fasciotomy versus limited fasciectomy. Plast Reconstr Surg. 129(2), 469- 
477.

9. Mafi R., Hindocha S., Khan W. (2012). Recent Surgical and Medical Advances in the treatment of Dupuytren's Disease - A Systematic Review of the Literature. Open Orthop J. 6, 77-82.

10. Peimer C.A., Blazar P., Coleman S., Kaplan F.T., Smith T., Tursi J.P., Cohen B., Kaufman G.J. \& Lindau T. (2013). Dupuytren Contracture Recurrence Following Treatment with Collagenase Clostridium Histolyticum (CORDLESS Study): 3-Year Data. J Hand Surg Am. 38(1), 12-22.

11. Dahlin L.B., Bainbridge C., Szczypa P.P., Cappelleri J.C., Guerin D. \& Gerber R.A. (2012). Current trends in the surgical management of Dupuytren's disease in Europe: the surgeon's perspective. Eur Orthop Traumatol. 3(1), 25-30.

12. Institute for Work \& Health. (2006). The QuickDASH outcome Measure Information for Users. Retreived Octomber 2013, from http:// www.dash.iwh.on.ca/system/files/quickdash_ info_2010.pdf.

13. The Swedish translation of the QuickDASH form. Available at: http://www.dash.iwh.on.ca/ system/files/translations/QuickDASH_Swedish. pdf. Accessed 01/10, 2013.

14. WHO: Obesity and overweight [Fact sheet No311]. (2012). Available at: http://www.who. int/mediacentre/factsheets/fs $311 / \mathrm{en} /$. Accessed 01/10, 2013.

15. Gummesson C., Ward M.M. \& Atroshi I. (2006). The shortened disabilities of the arm, shoulder and hand questionnaire (Quick DASH): validity and reliability based on responses within the full-length DASH. BMC MusculoskeletDisord. 7(44).

16. Dahlin L.B., Bainbridge C., Leclercq C., Gerber R.A., Guerin D., Cappelleri J.C., Szczypa P.P. \& Dias J. (2013). Dupuytren's disease presentation, referral pathwaysand resource utilisation in Europe: regional analysis of a surgeon surveyand patient chart review. Int J Clin Pract. 67(3), 261270.

17. Loos B., Puschkin V., Horch R.E. (2007) 50 years experience with Dupuytren's contracture in the Erlangen University Hospital - a retrospective analysis of 2919 operated hands from 1956 to
2006. BMC Musculoskelet Disord 8(60), 1-9

18. Dahlin L.B., Bainbridge C., Szczypa P.P., Cappelleri J.C., Guérin D \& Gerber R.A. (2012) Current trends in the surgical management of Dupuytren's disease in Europe: the surgeon's perspective. Eur OrthopTraumatol. doi: 10.1007/ s12570-012-0091-0

19. Rayan G.M. (1999) Clinical presentation and types of Dupuytren'sdisease. Hand Clin 7(15),87-96.

20. Barbosa R.I., da Silva Rodrigues E.K., Tamanini G., Marcolino A.M., Elui V.M., de Jesus Guirro R.R., Mazzer N. \& de Cássia Registro Fonseca M. (2012). Effectiveness oflow-level laser therapy for patients with carpal tunnel syndrome: design of a randomized single-blinded controlled trial. BMC Musculoskelet Disord.13(248). doi: 10.1186/1471-2474-13-248.

21. Cheung D.K., MacDermid J., Walton D. \& Grewal R. (2014) The construct validity and responsiveness of sensory tests in patients with carpal tunnel syndrome. Open Orthop J. 8,100-7. doi: 10.2174/1874325001408010100.

22. Jeong D.H. \& Kim C.H. (2014). The quantitative relationship between physical examinations and the nerve conduction of the carpal tunnel syndrome in patients with and without a diabetic polyneuropathy. Ann Rehabil. 38(1), 57-63. doi:10.5535/arm.2014.38.1.57.

23. 23.Jain N.B., Higgins L.D., Losina E., Collins J., Blazar P.E. \& Katz J.N. (2014). Epidemiology of musculoskeletal upper extremity ambulatory surgery in the United States. BMC Musculoskelet Disord. 8(15). doi: 10.1186/1471-2474-15-4.

24. 24.Toge Y., Nishimura Y., Basford J.R., Nogawa T., Yamanaka M., Nakamura T., Yoshida M., Nagano A. \& Tajima F. (2013). Comparison of the effects of flexion and extension of the thumb and fingers on the position and cross-sectional area of the median nerve. PLoS One. 8(12):e83565. doi: 10.1371/journal.pone.0083565.

25. Descatha A., Dale A.M., Franzblau A. \& Evanoff B. (2013). Natural history and predictors of longterm pain and function among workers with hand symptoms. Arch Phys Med Rehabil. 94(7), 12939. doi: 10.1016/j.apmr.2013.01.028.

26. Roll S.C., Evans K.D., Li X., Sommerich 
C.M. \& Case-Smith J. (2013). Importance of tissue morphology relative to patient reports of symptoms and functional limitations resulting from median nerve pathology. Am J Occup Ther. 67(1), 64-72. doi: 10.5014/ajot.2013.005785.

27. Ejiri S., Kikuchi S., Maruya M., Sekiguchi Y., Kawakami R. \& Konno S. (2012). Short-term results of endoscopic (Okutsu method) versus palmar incision open carpal tunnel release: a prospective randomized controlled trial. Fukushima J Med Sci. 58(1), 49-59.

28. de-la-Llave-Rincón A.I., Puentedura E.J. \& Fernández-de-las-Peñas C. (2012). New advances in the mechanisms and etiology of carpal tunnel syndrome. Discov Med. 13(72), 343-8.
29. Jeong J.S., Yoon J.S., Kim S.J., Park B.K., Won S.J., Cho J.M. \& Byun C.W. (2011). Usefulness ofultrasonography to predict response to injection therapy in carpal tunnelsyndrome. Ann Rehabil Med. 35(3),388-94. doi: 10.5535/ arm.2011.35.3.388.

30. Figures 1-11, personal archive Dr. Sebe Ioana Teona 